\title{
A Bayesian Joint Modeling Using Gaussian Linear Latent Variables for Mixed Correlated Outcomes with Possibility of Missing Values
}

\author{
Sayed Jamal Mirkamali \\ Shahid Beheshti University, Tehran, Iran \\ Mojtaba Ganjali* \\ Shahid Beheshti University, Tehran, Iran \\ m-ganjali@sbu.ac.ir
}

Received 12 December 2015

Accepted 5 May 2016

\begin{abstract}
This paper proposes a Bayesian approach for the analysis of mixed correlated nominal, ordinal and continuous outcomes with possibility of missing values using a variation of Markov Chain Monte Carlo (MCMC) method named Parameter Expanded and Reparamerized Metropolis Hastings (PX-RPMH) method. A general latent variable model is given and a Gibbs sampler is developed to incorporate PX-RPMH and Data Augmentation (DA) steps, to estimate parameters and to impute missing values. The performance of the algorithm is evaluated by some simulation studies. An application of the model to the foreign language attitude scale dataset is also enclosed.
\end{abstract}

Keywords: Mixed Data; Correlated Outcomes; Parameter Expansion; MCMC; Data Augmentation; Longitudinal Data.

2000 Mathematics Subject Classification: 62F15, 62H86, 62H25

\section{Introduction}

In many longitudinal studies and surveys the multivariate measurements with different scales are of the main interest. For example in Household Budget Surveys (HBS) there are a lot of questions about possession, level of income and consumption. Most of the time, there are some correlations between the responses. So, it is important to have statistical tools for analyzing such data. Many statistical procedures are developed to analyze different types of outcomes. The standard multivariate regression and cluster analysis for continuous outcomes, logistic regression and multinomial regression for nominal outcomes and cumulative probit and ordered logistic regression for ordinal outcomes are widely used to analyze these data. Olkin and Tate [31] developed a model for categorical and continuous outcomes named the general location model (GLOM). They modeled categorical and continuous outcomes by decomposing the joint model as a marginal multinomial

$\overline{{ }^{*} \text { Correspondence author, }}$ Professor of Statistics. 
distribution for categorical variables and a conditional multivariate normal distribution for continuous variables given the categorical variables. Some extensions of the GLOM are given in Liu and Rubin [24], Peng et al. [32] and Leon et al. [21]. A second method for joint modeling is to decompose the joint distribution as a multivariate marginal distribution for the continuous responses and a conditional distribution for categorical variables given the continuous variables. Cox and Wermuth [15] empirically examined the choice between these two methods. The third method uses simultaneous modeling of categorical and continuous variables to take into account the association between responses by the correlation between errors in the model. For more details of this approach see, for example, Heckman [18] in which a general model for simultaneously analyzing two mixed correlated responses is introduced. Catalano and Ryan [11] extended and used the model for clustered observations of discrete and continuous outcomes. The possibility of missing values for mixed discrete and continuous outcomes is studied by Fitzmaurice and Laird [17]. All the above references consider correlated nominal and continuous responses. A model for ordinal and continuous responses without considering any covariate effect is also presented by Poon and Lee [33]. Latent variable models are used by Bahrami Samani et al. [4] and the effect of missing values is studied by Bahrami Samani and Ganjali [5], Bahrami Samani et al. [6] and Bahrami Samani and Tahmasebinejad [7]. Bayesian models for analyzing mixed binary and continuous responses are presented by Liu et al. [26]. Zhang et al. [41] study a joint model for analyzing mixed nominal, ordinal and continuous data with possibility of missing values. A Bayesian joint analysis of mixed ordinal and skew continuous variables is presented by Teimourian et al. [38].

In this article, we propose a Bayesian joint model using latent variables for mixed correlated nominal, ordinal and continuous outcomes. We assume a multivariate normal distribution for latent variables and use a multivariate probit model for modeling nominal outcomes, an ordered probit model for ordinal outcomes and a multivariate regression model for continuous outcomes. To make a simultaneous analysis of these models we use a Parameter Expanded and Reparametrized Metropolis Hastings (PX-RPMH) algorithm within Gibbs sampler to get MCMC samples of parameters. In contrast to Zhang et al. [41], we use two approaches to improve the speed of the algorithm. First, we use different partitioning for cut points of ordinal latent variables. Second, we choose a jointly uniform prior for the covariance matrix and a conditionally independent sampler as our proposal in the Metropolis Hastings algorithm. We consider the possibility of missing values in each outcome and assume that the mechanism of missingness is at random (MAR). We also evaluate the sensitivity of our results when the missing mechanism is not at random (NMAR), (vide, Little and Rubin [23]).

This paper is organized as follows. In Section 2 we present the general mixed correlated model and the method of parameter estimation. In Section 3 we evaluate the model using some simulation studies. An application of our model is given in Section 4. Finally, the conclusions are presented in Section 5.

\section{Bayesian Modeling}

\subsection{The General Model for Analyzing Mixed Correlated Responses}

The idea of analyzing correlated binary outcomes using probit model was first proposed by Ashford and Sowden [3]. McFadden [29] generalized this idea to the Multinomial Probit (MNP) model using utility functions. A lot of work has been done about resolving the identifiability and generalizing of the MNP to the multivariate cases. See for example, McCulloch and Rossi [28], Chib and Greenberg 
[13], McCulloch et al. [27], Imai and Van Dyk [19], Xu and Craig [40], and Zhang et al. [41]. Here, we present notations for the Multivariate Probit (MVP) model.

Denote the $j$-th nominal outcome for subject $i$ by $N_{i j}$ for $j=1, \ldots, m_{1}$ where $N_{i j} \in\left\{0,1, \ldots, k_{j}-\right.$ $1\}$ and $k_{j}$ is the number of levels for $j$-th nominal outcome. For each nominal outcome we consider $k_{j}-1$ latent variables as $Z N_{i j, l}$ for $j=1, \ldots, m_{1}$ and $l=1, \ldots, k_{j}-1$. We relate the latent variables and observations by:

$$
N_{i j}=\left\{\begin{array}{l}
r \neq 0 \quad Z N_{i j, r}=\max _{1 \leq l \leq k_{j}-1} Z N_{i j, l}>0 \\
0 \quad \max _{1 \leq l \leq k_{j}-1} Z N_{i j, l} \leq 0 .
\end{array}\right.
$$

The latent variables correpond to each level of the nominal outcome are usually interpreted as the utility of that level in contrast to the utility of the first level of the nominal outcome.

Analyzing ordinal outcomes using probit model was first introduced by Aitchison and Silvey [1] and developed by Snell [35]. The identifiable multivariate ordered probit models studied by Nandram and Chen [30], Chen and Dey [12], Lawrence et al. [20], and Li and Schafer [22].

Denote the $j$-th ordinal outcome for subject $i$ by $O_{i j}$ for $j=1, \ldots, m_{2}$ where $O_{i j} \in\left\{1,2, \ldots, c_{j}\right\}$ and $c_{j}$ is the number of levels for ordinal outcome $j$. Let $Z O_{i j}$ be the latent variable associated with the $j$-th ordinal response where ordinal observations and latent variables are related as follow

$$
O_{i j}=r \Leftrightarrow \theta_{j, r-1}<Z O_{i j} \leq \theta_{j, r} \quad r=1, \ldots, c_{j},
$$

where $-\infty=\theta_{j, 0} \leq \theta_{j, 1} \leq \ldots \leq \theta_{j, c_{j}-1} \leq \theta_{j, c_{j}}=\infty$ with $\theta_{j, 1}=0$ for $j=1, \ldots, m_{2}$. Let $C_{i j}$ be $j-$ th continuous response of subject $i$ for $j=1, \ldots, m_{3}$.

Let $Y_{i}=\left(N_{i}^{T}, O_{i}^{T}, C_{i}^{T}\right)^{T}$ be $\left(m_{1}+m_{2}+m_{3}\right) \times 1$ vector of observations where $N_{i}^{T}=$ $\left(N_{i 1}, \ldots, N_{i m_{1}}\right), O_{i}^{T}=\left(O_{i 1}, \ldots, O_{i m_{2}}\right)$ and $C_{i}^{T}=\left(C_{i 1}, \ldots, C_{i m_{3}}\right)$ for subject $i, i=1, \ldots, n$. Now suppose that $Z_{i}=\left(Z N_{i}^{T}, Z O_{i}{ }^{T}, C_{i}{ }^{T}\right)^{T}$ be $q \times 1$ vector of continuous variables where $q=\Sigma_{j=1}^{m_{1}} k_{j}-$ $m_{1}+m_{2}+m_{3}, Z N_{i}^{T}=\left(Z N_{i 1}, \ldots, Z N_{i m_{1}}\right), Z N_{i j}=\left(Z N_{i j, 1}, \ldots, Z N_{i j, k_{j}-1}\right)$ for $j=1, \ldots, m_{1}$ and $Z O_{i}{ }^{T}=$ $\left(\mathrm{ZO}_{i 1}, \ldots, \mathrm{ZO}_{i m_{2}}\right)$.

Suppose that there are some covariates available for subject $i$, denoted by a $q \times p$ matrix of $X_{i}$. So we can write a latent variable model as

$$
Z_{i}=X_{i} \beta+\varepsilon_{i}
$$

where $\varepsilon_{i} \sim N_{q}(0, \Sigma)$ for $i=1, \ldots, n$. We take $Y=\left(Y_{1}, \ldots, Y_{n}\right), X=\left(X_{1}, \ldots, X_{n}\right)$ and $Z=\left(Z_{1}, \ldots, Z_{n}\right)$. Note that the design matrix $X_{i}$ and $\beta$ can be arranged so that the model can be used for analyzing both cross sectional and longitudinal data. For example, for analyzing longitudinal data with continuous outcomes suppose that $m_{1}=m_{2}=0$. To have a model with time varying covariates for $Z_{i}=\left(Z_{i, t_{1}}, Z_{i, t_{2}}, \ldots, Z_{i, t_{q}}\right)^{T}$ for time points $t_{1}, t_{2}, \ldots, t_{q}$, we can consider $X_{i}=\left(x_{i, t_{1}}^{T}, x_{i, t_{2}}^{T}, \ldots, x_{i, t_{q}}^{T}\right)^{T}$ and $\beta=\left(\beta_{1}, \ldots, \beta_{p}\right)^{T}$. If we need a model with fixed covariates and varying coefficients we can consider $X_{i}=I_{q} \otimes X_{i, t_{0}}$ and $\beta=\left(\beta_{1,1}, \beta_{1,2}, \ldots, \beta_{q, p_{0}}\right)^{T}$ where $X_{i, t_{0}}$ is the $1 \times p_{0}$ vector of fixed covariates for subject $i$ in time point $t_{0}$ and $p=q \times p_{0}$. Details can be found in Diggle et al. [16].

As noted by Chib and Greenberg [13] there are some restrictions on $\Sigma$ in context of identifiability of the latent model. If we partition $\Sigma$ according to $N O_{i}=\left(Z N_{i}, Z O_{i}\right)$ and $C_{i}$, we would have

$$
\Sigma=\left[\begin{array}{cc}
\Sigma_{N O} & \Sigma_{N O, C} \\
\Sigma_{C, N O} & \Sigma_{C}
\end{array}\right] .
$$


So, we need to take $\Sigma_{N O}$ as a correlation matrix. Therefore, $\Sigma$ is a covariance matrix with constraints that take diagonal elements corresponding to categorical outcomes to be equal to one. As a result of these restrictions, each of the off-diagonal elements of $\Sigma_{N O}$ belongs to $[-1,1]$.

\subsection{Priors}

For Bayesian inference, we need to specify priors for parameters in the models described in Section 2.1. We take the priors as

$$
\beta \sim N_{p}(\bar{\beta}, \bar{\Sigma})
$$

where $\bar{\beta}$ and $\bar{\Sigma}$ are hyperparameters that usually set to zero vector and $\sigma_{\beta}^{2} I$, respectively. We take

$$
p(\Theta) \propto \Pi_{j=1}^{m_{2}} 1_{\left(0 \leq \theta_{j, 2} \leq \ldots \leq \theta_{j, c_{j}-1}\right)}
$$

where $\Theta=\left(\theta_{1,2}, \ldots, \theta_{1, c_{1}-1}, \theta_{2,2}, \ldots, \theta_{m_{2} c_{m_{2}}-1}\right)$. The method of data augmentation introduced by Tanner and Wong [37] is useful when models include latent variables. This method is used by Albert and Chib [2] and Van Dyk and Meng [39] for Bayesian analysis of binary outcomes. Hence, we can obtain the posterior distribution of parameters through

$$
p(\beta, \Theta, \Sigma, Z \mid Y, X) \propto p(\beta) p(\Theta) p(\Sigma) \prod_{i=1}^{n} I N O_{i} \times \phi_{p}\left(Z_{i} ; X_{i} \beta, \Sigma\right)
$$

where $\phi_{p}(\cdot ; \mu, \Omega)$ represents the density function of $N_{p}(\mu, \Omega)$ distribution and $I N O_{i}$ is a compatibility indication which is equal to 1 if all latent variables $\left(Z N_{i}{ }^{T}, Z O_{i}{ }^{T}\right)^{T}$ are compatible with their corresponding observations $\left(N_{i}{ }^{T}, O_{i}{ }^{T}\right)$, and is equal to 0 , otherwise. More precisely, $I N O_{i}=I N_{i} \times I O_{i}$, $I N_{i}=\prod_{j=1}^{m_{1}} I N_{i j}, I O_{i}=\prod_{j=1}^{m_{2}} I O_{i j}$ and

$$
\begin{gathered}
I N_{i j}=1_{\left(N_{i j}=0, \max _{l} Z N_{i j, l}<0\right)}+\sum_{r=1}^{k_{j}-1} 1_{\left(N_{i j}=r, Z N_{i j, r}=\max _{l} Z N_{i j, l}>0\right)}, \\
I O_{i j}=\sum_{r=1}^{c_{j}} 1_{\left(O_{i j}=r, \theta_{j, r-1}<Z O_{i j} \leq \theta_{j, r}\right)} .
\end{gathered}
$$

To do Gibbs sampling we need full conditionals. It is straightforward to show that the posterior of coefficient parameters are distributed as

$$
\beta \mid \Theta, \Sigma, Z, Y, X \sim N_{p}(\tilde{\beta}, \tilde{\Sigma}),
$$

where $\tilde{\Sigma}=\left(\sum_{i=1}^{n} X_{i}^{T} \Sigma^{-1} X_{i}+\bar{\Sigma}^{-1}\right)^{-1}$ and $\tilde{\beta}=\tilde{\Sigma}\left(\sum_{i=1}^{n} X_{i}^{T} \Sigma^{-1} Z_{i}+\bar{\Sigma}^{-1} \bar{\beta}\right)$.

For the latent variables we have

$$
Z_{i j} \mid \beta, \Theta, \Sigma, Y, X,\left\{Z_{i j^{\prime}} ; j^{\prime} \neq j\right\} \sim T N\left(a_{i j}, s_{j}, B_{i j}\right), \quad j=1, \ldots, q-m_{3},
$$

where $a_{i j}=\mu_{i, j}-s_{j} \Sigma_{j,-j}^{-1}\left(Z_{i,-j}-\mu_{i,-j}\right), s_{j}=1 / \Sigma_{j j}^{-1}$ and $\mu_{i, j}$ is the $j-$ th element of $X_{i} \beta$ and $\mu_{i,-j}$ is the vector of $X_{i} \beta$ without $j$-th element and $\Sigma_{j j}^{-1}$ is the $(j, j)$-th element of $\Sigma^{-1}$ and $\Sigma_{j,-j}^{-1}$ is the $j$-th row of $\Sigma^{-1}$ without $j$-th element. Here, $T N\left(\mu, \sigma^{2}, B\right)$ is a truncated normal distribution over the 
interval $B$. The $B_{i j}$ interval is determined according to $Y_{i j}$. More specifically, for ordinal outcome $O_{i j}=r, B_{i j}=\left(\theta_{j, r-1}, \theta_{j, r}\right]$ and for nominal outcome $N_{i j}=0, B_{i j, l}=(-\infty, 0]$ for $l=1, \ldots, k_{j}-1$ and for $N_{i j}=r \neq 0, B_{i j, r}=\left(\max \left\{\max _{l \neq r}\left\{Z N_{i j, l}\right\}, 0\right\}, \infty\right)$ and $B_{i j, l}=\left(-\infty, Z N_{i j, r}\right)$ for $l \neq r$.

For cut points, as pointed out by Cowles [14] there is an approach to simulate the cut points by partitioning the parameters such that $\{Z, \Theta\}$ are simulated jointly, that is $p(Z, \Theta \mid \beta, \Sigma, Y, X) \propto$ $p(\Theta \mid \beta, \Sigma, Y, X) p(Z \mid \beta, \Theta, \Sigma, Y, X)$. As noted by Cowles [14], this strategy increases the speed of convergence of the algorithm. So we utilize its idea and generalize it for the multivariate case to make simulations according to the following posterior density:

$$
p(\Theta \mid \beta, \Sigma, Y, X) \propto \prod_{i=1}^{n} p\left(Z O_{i} \in B_{O_{i}, \Theta}\right)
$$

where $B_{O_{i}, \Theta}=\prod_{j=1}^{m_{2}}\left(\theta_{j, O_{i j}-1}, \theta_{j, O_{i j}}\right]$ and $Z O_{i} \sim N_{m_{2}}\left(X_{i, O} \beta, \Sigma_{O}\right)$ and $X_{i, O}$ and $\Sigma_{O}$ are the design matrix and the covariance matrix corresponding to the ordinal latent variables, respectively. Simulation is implemented using a Metropolis-Hastings step as below:

1. Initialize $\Theta^{(0)}=\left(\theta_{1,2}^{(0)}, \theta_{1,3}^{(0)}, \ldots, \theta_{m_{2}, c_{m_{2}}-1}^{(0)}\right)$ according to the results of ordinal probit models and set $\sigma_{M H}=0.05 / \bar{c}$ where $\bar{c}=\sum_{j=1}^{m_{2}} c_{j}$, as a rule of thumb for candidate hyperparameter to get appropriate acceptance rates.

2. For $j=1, \ldots, m_{2}$ and $r=2, \ldots, c_{j}-1$ generate

$$
\theta_{r, j}^{*} \sim T N\left(\theta_{r, j}^{(t-1)}, \sigma_{M H}^{2},\left(\theta_{r-1, j}^{*}, \theta_{r+1, j}^{(t-1)}\right]\right) .
$$

3. Set $\Theta^{(t)}=\Theta^{(t-1)}$ with probability of $1-R$ and set $\Theta^{(t)}=\Theta^{*}$ with probability of $R$ as

$$
\begin{aligned}
R & =\min \left\{1, \prod_{i=1}^{n} \frac{p\left(Z O_{i} \in B_{O_{i}, \Theta^{*}}\right)}{p\left(Z O_{i} \in B_{\left.O_{i}, \Theta^{(t-1)}\right)}^{(t-1)}\right.}\right. \\
& \left.\times \prod_{j=1}^{m_{2}} \prod_{r=2}^{c_{j}-1} \frac{\Phi\left(\left(\theta_{j, r+1}^{(t-1)}-\theta_{j, r}^{(t-1)}\right) / \sigma_{M H}\right)-\Phi\left(\left(\theta_{j, r-1}^{*}-\theta_{j, r}^{(t-1)}\right) / \sigma_{M H}\right)}{\Phi\left(\left(\theta_{j, r+1}^{*}-\theta_{j, r}^{*}\right) / \sigma_{M H}\right)-\Phi\left(\left(\theta_{j, r-1}^{(t-1)}-\theta_{j, r}^{*}\right) / \sigma_{M H}\right)}\right\} .
\end{aligned}
$$

4. Increase $t=t+1$ and repeat the steps (2) and (3) until an adequate number of samples are obtained.

For an unconstrained covariance Box and Tiao [9] presented the Jeffreys prior as $p(\Sigma) \propto$ $|\Sigma|^{-(q+1) / 2}$ and the inverse Wishart distribution as a conjugate prior. However, for constrained covariances there is no standard distribution available as a prior. Different priors and simulation procedures proposed by McCulloch and Rossi [28], Chib and Greenberg [13], Barnard et al. [8], Imai and Van Dyk [19], and Burgette and Nordheim [10]. Barnard et al. [8] studied marginal uniform priors and jointly uniform priors for correlation matrices. In this paper we specify a jointly uniform prior for $\Sigma$ constrained to be a positive definite matrix with some diagonal elements equal to one, i.e.

$$
p(\Sigma) \propto 1_{(\Sigma \text { is positive definite and diagonals of discrete variables are equal to one }) .}
$$

Obviously the posterior of $\Sigma$ cannot be obtained conveniently. Hence, we use the PX-RPMH algorithm within Gibbs sampler to simulate covariance matrix $\Sigma$. Details are given in the next subsection.

\subsection{The PX-RPMH Algorithm for Simulating Covariance Matrix}

To make simulations from $\Sigma$, we use the method of parameter expansion which is implemented by Liu and Daniels [25] and Liu et al. [26]. The method includes four steps of (a) parameter expansion, 
(b) transformation, (c) defining candidate prior and candidate posterior and (d) simulation according to Metropolis-Hastings. Details of these steps are given in the following.

(a) In this step we need to expand $\Sigma$ into a less constrained matrix $\Sigma_{\circ}$ which is just a positive definite matrix, and to define a reduction operator as $(\beta, \Sigma)=\operatorname{Red}\left(\beta, \Sigma_{\circ}\right)=\left(\beta, D_{\circ}^{-1} \Sigma_{\circ} D_{\circ}^{-1}\right)$ where $D_{\circ}$ is the matrix of square root of diagonal elements of $\Sigma_{\circ}$ with replacement of diagonal elements corresponding to continuous outcomes with one.

(b) This step is to transform $\left\{Z_{i}, \Sigma\right\}$ into $\left\{Z_{i}^{*}, \Sigma_{\circ}\right\}$ according to the following one-to-one mapping:

$$
\left\{\begin{array}{l}
Z_{i}=X_{i} \beta+D_{\circ}^{-1} Z_{i}^{*} \\
\Sigma=D_{\circ}^{-1} \Sigma_{\circ} D_{\circ}^{-1}
\end{array} \quad i=1, \ldots, n,\right.
$$

where $\sum_{i=1}^{n} Z_{i j}^{* 2}=1$ for any $j=1, \ldots, q$. These restrictions are needed to make the transformation one-to-one mapping. Note that draws of $Z_{i}$ and $\beta$ implicitly correspont to draws of $Z_{i}^{*}$ and $D_{\circ}$, because

$$
\sum_{i=1}^{n}\left(Z_{i j}-x_{i j}^{\prime} \beta\right)^{2}=\left(D_{\circ}^{-1}\right)_{j j}^{2} \sum_{i=1}^{n} Z_{i j}^{* 2}=\left(D_{\circ}^{-1}\right)_{j j}^{2}, \quad j=1, \ldots, q
$$

where $x_{i j}^{\prime}$ is the $j$-th row of $X_{i}$ and $\left(D_{\circ}^{-1}\right)_{j j}$ is the $j$-th diagonal element of $D_{\circ}^{-1}$.

(c) Here, we find the candidate prior given by $\pi(\Sigma) \propto|\Sigma|^{-\frac{q+1}{2}}$. It can be verified by using (2.5) that

$$
\pi\left(\Sigma_{\circ} \mid Z^{*}, \beta\right) \propto\left|\Sigma_{\circ}\right|^{-\frac{n+q+1}{2}} \operatorname{etr}\left\{-\frac{1}{2} S \Sigma_{\circ}^{-1}\right\},
$$

where $\operatorname{etr}\{\cdot\}=\exp \{\operatorname{trace}(\cdot)\}, S=\sum_{i=1}^{n} Z_{i}^{*} Z_{i}^{* T}$ and $Z_{i}^{*}=D_{\circ}\left(Z_{i}-X_{i} \beta\right)$, that is $\Sigma_{\circ} \mid Z^{*}, \beta$ is distributed as inverse Wishart with $n$ degrees of freedom and the scale matrix of $S$.

(d) This step is to draw $\Sigma_{\circ}^{*}$ according to the distribution of $\Sigma_{\circ} \mid Z^{*}, \beta$ and translating it back to $\Sigma^{*}=D_{\circ}^{*-1} \Sigma_{\circ}^{*} D_{\circ}^{*-1}$ and accepting the candidate $\Sigma^{*}$ using a Metopolis-Hastings step with acceptance rate

$$
\alpha=\min \left\{1, \exp \left(\frac{q+1}{2}\left(\log \left|\Sigma^{*}\right|-\log \left|\Sigma^{(t)}\right|\right)\right)\right\}
$$

at iteration $t+1$.

More details and proofs can be found in Liu and Daniels [25] and Liu et al. [26].

\subsection{Handling Missing Values}

When there are some missing values in $Y$, we partition it as $Y=\left(Y_{o b s}, Y_{m i s}\right)$ where $Y_{o b s}$ is the observed part of $Y$ and $Y_{m i s}$ is the missing part of $Y$. In this article we assume the mechanism of missingness to be at random. That is $p\left(M_{i j} \mid Y\right)=p\left(M_{i j} \mid Y_{o b s}\right)$ where $M_{i j}$ is equal to one if $Y_{i j}$ is missed and is equal to zero if $Y_{i j}$ is observed. Therefore, we can use the Data Augmentation Modeling described by distribution given in (2.3) to make draws of missing latent variables according to the model described in (2.1) for $Y_{o b s}$. So, all of the parametric models remain valid except that $Z_{i j} \mid \beta, \Theta, \Sigma, Y, X,\left\{Z_{i j^{\prime}} ; j^{\prime} \neq j\right\}$ is no longer truncated. It should be noted that when there is no missing values for continuous variables we just make draws of the latent variables of categorical variables. 
However, when there are some missing values in continuous variables one should treat them as latent variables, namely $Z C_{i j} \mathrm{~s}$, which correspond to $C_{i j}$ s that have missing values.

\subsection{Initializations and Tunings}

At the first step of MCMC we need some initial values for parameters. In this study we set them randomly. However, we recommend the use of the output of an ordered logistic regression model for initial values of cut points, pairwise sample association coefficients for initial values of correlation matrix and the output of a linear regression model for initial values of coefficients.

\section{Simulation Study}

\subsection{Data Generation}

We use the following simulated example to illustrate the Bayesian model and the MCMC algorithm proposed in section 2 for analyzing mixed nominal, ordinal and continuous correlated outcomes. For $n=300$ individuals we take a nominal outcome $N$ with three levels, an ordinal outcome $O$ with four levels and a continuous outcome $Z$. We also generated three covariates from $U(-0.5,0.5)$ and set the first column of $X_{i}$ to one, which means $p=4$. So the latent variables model can be expressed as

$$
Z_{i}=X_{i} \beta+\varepsilon_{i} \quad i=1, \ldots, n,
$$

where $Z_{i}=\left(Z N_{i 1}, Z N_{i 2}, Z O_{i}, C_{i}\right)$ and $\varepsilon_{i} \sim N_{4}(0, \Sigma)$. We take $\beta=(1,-2,0,1)^{T}$ and $\left(\theta_{2}, \theta_{3}\right)=(0.5,1)$. According to restrictions on the covariance matrix, we have

$$
\Sigma=\left[\begin{array}{cccc}
1 & \sigma_{12} & \sigma_{13} & \sigma_{14} \\
\sigma_{12} & 1 & \sigma_{23} & \sigma_{24} \\
\sigma_{13} & \sigma_{23} & 1 & \sigma_{34} \\
\sigma_{14} & \sigma_{24} & \sigma_{34} & \sigma_{44}
\end{array}\right]
$$

Here, we take $\sigma_{44}=2$ and $\sigma_{i j}=0.5$ for $i \neq j$.

\subsubsection{Evaluation of Parameter Estimates}

Suppose that the parameter space is $\Xi=\{\beta, \Theta, \Sigma\}$ and $\xi \in \Xi$ is any parameter of the model. To make an estimation of $\xi$ we iterated the MCMC algorithm for $n_{\text {iter }}=75$ times with $n_{\text {gibbs }}=2000$ Gibbs samples and $n_{\text {burn }}=500$ burning samples and the thinning number $n_{\text {thin }}=3$ to get $n_{\text {mcmc }}=500$ samples of posterior densities denoted by $\left\{\xi_{i, j}\right\}$ for $i=1, \ldots, n_{m c m c} ; j=1, \ldots, n_{i t e r}$. We estimated the parameter $\xi$ and its standard error by $\hat{\mu}_{\xi}$ and $\hat{\sigma}_{\xi}$, respectively as $\hat{\mu}_{\xi}=\sum_{j=1}^{n_{\text {iter }}} \bar{\xi}_{j} / n_{\text {iter }}$; and $\hat{\sigma}_{\xi}=$ $\sqrt{\sum_{j=1}^{n_{i t e r}}\left(\bar{\xi}_{j}-\overline{\bar{\xi}}_{j}\right) /\left(n_{\text {iter }}-1\right)}$; where $\bar{\xi}_{j}=\sum_{i=1}^{n_{\text {mcmc }}} \xi_{i, j} / n_{m c m c}$ for $j=1, \ldots, n_{\text {iter }}$.

The results are given in Table 1. In this table we see that MCMC sampler is successful to estimate $\beta_{0}, \beta_{1}, \beta_{2}, \beta_{3}$ and the cut points. However, there is an underestimation regarding the covariances by a factor of $10 \%$ for sample size $n=300$. The standard error of parameters are relatively low, with respect to the magnitude of true values, that show a measure of goodness of convergence. The standard errors reduce as the sample size increases. 
Table 1. Summary of 75 iterations of MCMC

\begin{tabular}{cccccc}
\hline \multirow{2}{*}{ Parameters } & True & \multicolumn{2}{c}{$n=300$} & \multicolumn{2}{c}{$n=1000$} \\
\cline { 3 - 6 } & Values & Est. & S.E. & Est. & S.E. \\
\hline$\beta_{0}$ & 1.0 & 1.007 & 0.072 & 0.997 & 0.030 \\
$\beta_{1}$ & -2.0 & -1.998 & 0.145 & -2.002 & 0.074 \\
$\beta_{2}$ & 0.0 & -0.023 & 0.110 & -0.005 & 0.053 \\
$\beta_{3}$ & 1.0 & 1.009 & 0.125 & 1.006 & 0.078 \\
$\theta_{2}$ & 0.5 & 0.503 & 0.070 & 0.498 & 0.034 \\
$\theta_{3}$ & 1.0 & 1.010 & 0.091 & 0.991 & 0.040 \\
$\sigma_{12}$ & 0.5 & 0.458 & 0.112 & 0.483 & 0.059 \\
$\sigma_{13}$ & 0.5 & 0.461 & 0.106 & 0.492 & 0.071 \\
$\sigma_{14}$ & 0.5 & 0.464 & 0.166 & 0.496 & 0.092 \\
$\sigma_{23}$ & 0.5 & 0.473 & 0.117 & 0.480 & 0.069 \\
$\sigma_{24}$ & 0.5 & 0.472 & 0.163 & 0.499 & 0.082 \\
$\sigma_{34}$ & 0.5 & 0.505 & 0.103 & 0.501 & 0.051 \\
$\sigma_{44}$ & 2.0 & 2.074 & 0.175 & 2.020 & 0.085 \\
\hline
\end{tabular}

\subsection{Prediction and Validation}

We used a 5-fold cross validation for a sample of size $n=300$ that is we partitioned the sample into 5 subsamples and used four subsamples to estimate the parameters and one subsample to predict the observations jointly and to find the accuracy of predictions. We measured the accuracy of nominal and ordinal variables by the percentage of correct predictions and the accuracy of continuous variable by mean square prediction error (MSPE) defined as $M S P E=\frac{1}{m} \sum_{i=1}^{m}\left(\hat{W}_{i}-W_{i}\right)^{2}$, where $\hat{W}_{i}$ is the predicted value for observation $W_{i}$ and $m=n / 5$, the number of predictions. According to Table 2 , on average $67 \%$ of nominal outcomes predicted exactly. This index for ordinal outcome is about $50 \%$ while this outcome has 4 levels and the correct predictions of a random guess would be only $25 \%$. The positive correlations between predictions and the true values show that the predictions are in the same direction of the true values. The MSPE of continuous variable is near the true variance showing that the model does not inflate the variations. It should be noted that the accuracy of the model highly depends on the variations of predictors and the variations of errors, that is the accuracy of cross validation will increase when variation of predictors increase and it will decrease as the variations of errors increase.

Table 2. Cross validation summary

\begin{tabular}{cccc}
\hline Variable & Accuracy $(\%)$ & Correlation & MSPE \\
\hline Nominal & 67.41 & - & - \\
Ordinal & 49.72 & 0.48 & - \\
Continuous & - & 0.41 & 2.03 \\
\hline
\end{tabular}

\subsection{The Effect of Missing at Random Assumption}

To make an evaluation of the effect of missingness we generated a sample of size $n=300$, and then removed some of the outcomes as missing values according to three different scenarios: 
Table 3. Summary of 75 iterations of MCMC with MAR values

\begin{tabular}{cccccccc}
\hline \multirow{2}{*}{ Parameters } & True & \multicolumn{2}{c}{ M1 } & \multicolumn{2}{c}{ M2 } & \multicolumn{2}{c}{ M3 } \\
\cline { 3 - 8 } & Values & Est. & S.E. & Est. & S.E. & Est. & S.E \\
\hline$\beta_{0}$ & 1.0 & 1.002 & 0.062 & 0.997 & 0.073 & 0.990 & 0.060 \\
$\beta_{1}$ & -2.0 & -2.032 & 0.151 & -2.008 & 0.175 & -2.044 & 0.150 \\
$\beta_{2}$ & 0.0 & -0.018 & 0.121 & 0.017 & 0.142 & -0.024 & 0.152 \\
$\beta_{3}$ & 1.0 & 1.011 & 0.149 & 1.041 & 0.131 & 1.055 & 0.156 \\
$\theta_{2}$ & 0.5 & 0.506 & 0.066 & 0.490 & 0.068 & 0.488 & 0.070 \\
$\theta_{3}$ & 1.0 & 1.009 & 0.089 & 0.999 & 0.082 & 0.979 & 0.091 \\
$\sigma_{12}$ & 0.5 & 0.429 & 0.118 & 0.451 & 0.121 & 0.423 & 0.144 \\
$\sigma_{13}$ & 0.5 & 0.462 & 0.106 & 0.452 & 0.173 & 0.486 & 0.170 \\
$\sigma_{14}$ & 0.5 & 0.465 & 0.165 & 0.464 & 0.145 & 0.470 & 0.160 \\
$\sigma_{23}$ & 0.5 & 0.463 & 0.107 & 0.444 & 0.181 & 0.480 & 0.160 \\
$\sigma_{24}$ & 0.5 & 0.451 & 0.154 & 0.469 & 0.138 & 0.460 & 0.142 \\
$\sigma_{34}$ & 0.5 & 0.470 & 0.106 & 0.506 & 0.103 & 0.482 & 0.086 \\
$\sigma_{44}$ & 2.0 & 2.065 & 0.199 & 2.059 & 0.159 & 2.072 & 0.143 \\
\hline
\end{tabular}

M1. Missing values only correspond to the continuous variable with

$$
p\left(M C_{i j}=1 \mid O_{i j}=o_{i j}\right)= \begin{cases}0.4 & o_{i j}=4 \\ 0.1 & o_{i j} \neq 4\end{cases}
$$

where $M C_{i j}$ indicates missing status of $C_{i j}$.

M2. Missing values only correspond to the ordinal variable with

$$
p\left(M O_{i j}=1 \mid N_{i j}=n_{i j}\right)= \begin{cases}0.5 & n_{i j}=0 \\ 0.1 & n_{i j} \neq 0\end{cases}
$$

where $M O_{i j}$ indicates missing status of $O_{i j}$.

M3. Missing values only correspond to the nominal variable with

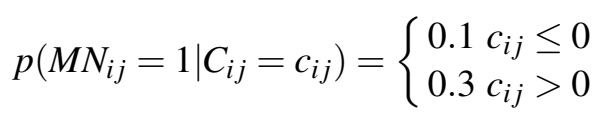

where $M N_{i j}$ indicates missing status of $N_{i j}$.

In all three scenarios we have one variable with missing values and other variables are observed for all individuals. In each scenario, the probabilities of missingness are chosen such that the rate of missingness is about $25 \%$. The estimated parameters and standard errors reported in Table 3 show that the algorithm is successful even if there are MAR values in the dataset.

\subsection{Sensitivity to Non-Ignorable Missing Values}

To make an evaluation of the effect of missing not at random mechanism we generated a sample of size $n=300$, and then replaced some of the responses of continuous variable with missing values according to the following two different scenarios:

M4. Missing values correspond to the continuous variable with

$$
p\left(M C_{i j}=1 \mid C_{i j}=c_{i j}\right)= \begin{cases}0.4 & c_{i j} \leq 0 \\ 0.1 & c_{i j}>0\end{cases}
$$


Table 4. Summary of 75 iterations of MCMC for different patterns of missing values

\begin{tabular}{|c|c|c|c|c|c|c|c|c|c|}
\hline \multirow{2}{*}{$\begin{array}{l}\text { Param- } \\
\text { eters }\end{array}$} & \multirow{2}{*}{$\begin{array}{l}\text { True } \\
\text { Values }\end{array}$} & \multicolumn{2}{|c|}{ No Missing } & \multicolumn{2}{|c|}{ M1 (MAR) } & \multicolumn{2}{|c|}{ M4 (NMAR) } & \multicolumn{2}{|c|}{ M5 (NMAR) } \\
\hline & & Est. & S.E. & Est. & S.E. & Est. & S.E & Est. & S.E. \\
\hline$\beta_{0}$ & 1.0 & 1.007 & 0.072 & 1.007 & 0.068 & 0.928 & 0.065 & 1.247 & 0.076 \\
\hline$\beta_{1}$ & -2.0 & -1.998 & 0.145 & -2.036 & 0.168 & -2.061 & 0.178 & -1.839 & 0.285 \\
\hline$\beta_{2}$ & 0.0 & -0.023 & 0.110 & -0.020 & 0.128 & -0.009 & 0.118 & 0.001 & 0.121 \\
\hline$\beta_{3}$ & 1.0 & 1.009 & 0.125 & 1.008 & 0.147 & 1.026 & 0.143 & 0.906 & 0.194 \\
\hline$\theta_{2}$ & 0.5 & 0.503 & 0.070 & 0.502 & 0.065 & 0.457 & 0.066 & 0.612 & 0.082 \\
\hline$\theta_{3}$ & 1.0 & 1.010 & 0.091 & 1.008 & 0.085 & 0.936 & 0.079 & 1.151 & 0.101 \\
\hline$\sigma_{12}$ & 0.5 & 0.458 & 0.112 & 0.433 & 0.117 & 0.352 & 0.142 & 0.478 & 0.292 \\
\hline$\sigma_{13}$ & 0.5 & 0.461 & 0.106 & 0.455 & 0.107 & 0.431 & 0.117 & 0.433 & 0.301 \\
\hline$\sigma_{14}$ & 0.5 & 0.464 & 0.166 & 0.459 & 0.167 & 0.457 & 0.193 & 0.217 & 0.202 \\
\hline$\sigma_{23}$ & 0.5 & 0.473 & 0.117 & 0.462 & 0.109 & 0.429 & 0.131 & 0.423 & 0.314 \\
\hline$\sigma_{24}$ & 0.5 & 0.472 & 0.163 & 0.444 & 0.154 & 0.481 & 0.169 & 0.212 & 0.197 \\
\hline$\sigma_{34}$ & 0.5 & 0.505 & 0.103 & 0.470 & 0.107 & 0.508 & 0.114 & 0.254 & 0.110 \\
\hline$\sigma_{44}$ & 2.0 & 2.074 & 0.175 & 2.053 & 0.199 & 2.195 & 0.197 & 1.504 & 2.664 \\
\hline
\end{tabular}

M5. Missing values correspond to the continuous variable with

$$
p\left(M C_{i j}=1 \mid C_{i j}=c_{i j}\right)= \begin{cases}1 & c_{i j} \leq 0 \\ 0 & c_{i j}>0\end{cases}
$$

The estimated parameters in Table 4 show that the true values of parameters are within two standard deviation intervals. However, the biases of parameters raise in non-ignorable missing patterns especially for those of the covariance parameters which are directly connected to variables containing missing values. The analysis of standard errors shows that there is an increase in error of estimations, especially for the covariance parameters, when the probability of non-ignorable missingness increases.

\section{Application}

As an application of our model we used the Foreign Language Attitude Scale (FLAS) data which was analyzed by Schafer [34] using GLOM. It contains $n=279$ samples of students who enrolled in foreign language courses at the Pennsylvania State university. We investigated the association between the kind of foreign language studied (LAN), Modern Language Aptitude Test (MLAT), and the age of students (AGE) with the FLAS variable as a predictor. Table 5 presents some descriptive statistics of the dataset.

We start by a saturated model with separate coefficients for each latent variable. Due to different sample sizes for levels of the nominal outcome we used a mildly informative prior regarding to coefficients of nominal latent variables as $\left(\beta_{0, Z N_{1}}, \beta_{1, Z N_{1}}, \ldots, \beta_{1, Z N_{3}}\right) \sim N(0, I)$ and non-informative priors for other coefficients. The results of the MCMC algorithm are presented in Table 6 . The outputs of the saturated model show that the FLAS score has no effect on the latent variables of Spanish and German languages. However, there is a negative effect on the latent variable of Russian language. It means that those who get more FLAS scores are less likely to choose Russian language. Obviously, the posterior distributions of the latent variables of Spanish and German languages are very similar. Also the negative coefficient corresponding to ordinal latent variable means that those who get more FLAS score are less likely to be older. The estimated coefficients for continuous outcomes are similar to the standard regression model $M L A T_{i}=18+0.08 F L A S_{i}$. Because there are 
Table 5. Summary of variables in foreign language attitude scale analysis

\begin{tabular}{llllll}
\hline Variable & Description & Categories & Percent & Missing & Latents \\
\hline LAN & Foreign & French & $24.0 \%$ & $0.0 \%$ & - \\
& language & Spanish & $28.0 \%$ & & $Z N_{1}$ \\
& studied & German & $40.9 \%$ & & $Z N_{2}$ \\
& & Russian & $7.1 \%$ & & $Z N_{3}$ \\
\multirow{5}{*}{ AGE } & Age group & $<20$ & $46.3 \%$ & $3.9 \%$ & $Z O$ \\
& & $20-21$ & $43.3 \%$ & & \\
& & $\geq 22$ & $10.4 \%$ & & \\
MLAT & Modern & & & & \multirow{2}{*}{$17.6 \%$} \\
& Language & & & & \\
& Aptitude Test & & & & \\
& & & & $0.0 \%$ & - \\
FLAS & Foreign & & & & \\
& Language & & & & \\
& Attitude Scale & & & & \\
\hline
\end{tabular}

many non-significant coefficients in the saturated model, we consider a reduced model by assuming $\beta_{1, Z N_{1}}=\beta_{1, Z N_{2}}=0$. The results of the reduced model show a substantial reduction in standard error of parameters. For comparison of the full model and the reduced model we use the DIC index (Spiegelhalter et al. [36]). The difference between DICs is quite large showing that the fit of the reduced model is better than that of the full model. The positive correlation between latent variables of Spanish and German languages $\left(\sigma_{Z N_{1}, Z N_{2}}=0.811\right)$ shows that the utilities of these choices are in the same direction. The negative correlations between the latent variables of German and Russian languages with AGE group show that younger students are expected to choose German and Russian courses. The positive covariance of $\sigma_{Z N_{3}, Z C}$ and the negative covariance of $\sigma_{Z O, Z C}$ suggest that the students who choose Russian language and younger students are expected to get more MLAT scores.

\section{Conclusions}

We have developed a joint model for correlated mixed outcomes using MCMC approach. The results of simulations show that our method is successful in estimation of parameters of the model. A cross validation analysis of simulated data with MAR values shows that the method is reliable regarding parameter estimation and imputations. Further investigations about NMAR mechanism show that our method is not successful about parameter estimation and this type of missingness will raise standard errors slightly. As an application of our method we studied the foreign language aptitude scale dataset. Also, the thetracoric correlations for this dataset were investigated.

It should be noted that the MVP model is sensitive to zero counts in the cross tabulation of nominal outcomes and non-informative priors for coefficients may result in divegence of MCMC algorithm. So, we recommend to put informative priors for those parameters.

Our extensive studies suggest that our Bayesian methodology can be used effectively in the studies where joint analysis of a combination of correlated nominal, ordinal and continuous outcomes is of the main interests. It is obvious that our method can be used for longitudinal studies with arbitrary correlation matrices. 
Table 6. Estimated parameters of the latent variables for FLAS dataset

\begin{tabular}{|c|c|c|c|c|}
\hline \multirow{2}{*}{ Parameters } & \multicolumn{2}{|c|}{ Saturated Model } & \multicolumn{2}{|c|}{ Reduced Model } \\
\hline & Estimates & S.E. & Estimates & S.E. \\
\hline$\beta_{0, Z N_{1}}$ & 0.181 & 0.355 & $0.137 *$ & 0.058 \\
\hline$\beta_{1, Z N_{1}}$ & 0.001 & 0.006 & - & - \\
\hline$\beta_{0, Z N_{2}}$ & 0.019 & 0.378 & $0.213^{*}$ & 0.059 \\
\hline$\beta_{1, Z N_{2}}$ & 0.003 & 0.004 & - & - \\
\hline$\beta_{0, Z N_{3}}$ & $-1.278^{*}$ & 0.510 & $-0.390 *$ & 0.073 \\
\hline$\beta_{1, Z N_{3}}$ & -0.004 & 0.005 & $-0.013^{*}$ & 0.001 \\
\hline$\beta_{0, Z O}$ & $1.339 *$ & 0.410 & $0.999 *$ & 0.407 \\
\hline$\beta_{1, Z O}$ & $-0.015^{*}$ & 0.006 & $-0.010^{*}$ & 0.005 \\
\hline$\beta_{0, Z C}$ & $16.643^{*}$ & 2.409 & $17.220 *$ & 2.293 \\
\hline$\beta_{1, Z C}$ & $0.092 *$ & 0.028 & $0.086^{*}$ & 0.027 \\
\hline$\theta_{2}$ & $1.507 *$ & 0.144 & $1.431 *$ & 0.093 \\
\hline$\sigma_{Z N_{1}, Z N_{2}}$ & $0.811^{*}$ & 0.302 & $0.822 *$ & 0.160 \\
\hline$\sigma_{Z N_{1}, Z N_{3}}$ & $-0.698 *$ & 0.224 & -0.496 & 0.246 \\
\hline$\sigma_{Z N_{1}, Z O}$ & -0.077 & 0.218 & 0.050 & 0.112 \\
\hline$\sigma_{Z N_{1}, Z C}$ & 0.459 & 0.781 & -0.195 & 0.511 \\
\hline$\sigma_{Z N_{2}, Z N_{3}}$ & $-0.754 *$ & 0.154 & 0.007 & 0.071 \\
\hline$\sigma_{Z N_{2}, Z O}$ & -0.167 & 0.150 & $-0.113 *$ & 0.076 \\
\hline$\sigma_{Z N_{2}, Z C}$ & $1.131 *$ & 0.400 & 0.427 & 0.408 \\
\hline$\sigma_{Z N_{3}, Z O}$ & -0.221 & 0.116 & $-0.295^{*}$ & 0.069 \\
\hline$\sigma_{Z N_{3}, Z C}$ & -0.781 & 0.642 & $1.117 *$ & 0.439 \\
\hline$\sigma_{Z O, Z C}$ & $-1.155^{*}$ & 0.478 & $-1.189 *$ & 0.512 \\
\hline$\sigma_{Z C}^{2}$ & $41.771 *$ & 9.991 & $40.137^{*}$ & 3.543 \\
\hline DIC & 2979.33 & & 2904.89 & \\
\hline
\end{tabular}

\section{References}

[1] Aitchison, J. and Silvey, S. D., The generalization of probit analysis to the case of multiple responses. Biometrika, 44(1-2)(1957) 131-140.

[2] Albert, J. H. and Chib, S., Bayesian analysis of binary and polychotomous response data. Journal of the American statistical Association, 88(422)(1993), 669-679.

[3] Ashford, J. R. and Sowden, R. R., Multi-variate probit analysis. Biometrics(1970) 535-546.

[4] Bahrami Samani, E., Ganjali, M. and Khodaddadi, A., A latent variable model for mixed continuous and ordinal responses. Journal of Statistical Theory and Applications, 7(3)(2008) 337-349.

[5] Bahrami Samani, E. and Ganjali, M., A Multivariate Latent Variable Model for Mixed Data from Continuous and Ordinal Responses with Possibility of Missing Responses. Appl. Appl. Math.: Int. J, 5(10)(2010) 1564-1584.

[6] Bahrami Samani, E., Ganjali, M. and Eftekhari, S., A latent variable model for mixed continuous and ordinal responses with nonignorable missing responses: Assessing the local influence via covariance structure. Sankhya B, 72(1)(2010) 38-57.

[7] Bahrami Samani, E. and Tahmasebinejad, Z., Joint Modelling of Mixed Correlated Nominal, Ordinal and Continuous Responses. Journal of Statistical Research, 45(1)(2011) 37-47.

[8] Barnard, J., McCulloch, R. and Meng, X. L., Modeling covariance matrices in terms of standard deviations and correlations, with application to shrinkage. Statistica Sinica, 10(4)(2000) 1281-1312.

[9] Box, G. E. and Tiao, G. C., Bayesian inference in statistical analysis. (Addison-Wesely publishing company, 1973).

[10] Burgette, L. F. and Nordheim, E. V., The trace restriction: An alternative identification strategy for the Bayesian multinomial probit model. Journal of Business \& Economic Statistics, 30(3)(2012) 404-410. 
[11] Catalano, P. J. and Ryan, L. M., Bivariate latent variable models for clustered discrete and continuous outcomes. Journal of the American Statistical Association, 87(419)(1992) 651-658.

[12] Chen, M. H. and Dey, D. K., Bayesian analysis for correlated ordinal data models. Biostatistics Basel, 5(2000) 133-158.

[13] Chib, S. and Greenberg, E., Analysis of multivariate probit models. Biometrika, 85(2)(1998) 347-361.

[14] Cowles, M. K., Accelerating Monte Carlo Markov chain convergence for cumulative-link generalized linear models. Statistics and Computing, 6(2)(1996) 101-111.

[15] Cox, D. R. and Wermuth, N., Response models for mixed binary and quantitative variables. Biometrika, 79(3)(1992) 441-461.

[16] Diggle, P., Heagerty, P., Liang, K. Y. and Zeger, S., Analysis of longitudinal data. (Oxford University Press, New York, 2002).

[17] Fitzmaurice, G. M. and Laird, N. M., Regression models for mixed discrete and continuous responses with potentially missing values. Biometrics(1997) 110-122.

[18] Heckman, J. J., Dummy endogenous variables in a simultaneous equation system. Econometrica, 46(4)(1978) 931-959.

[19] Imai, K. and Van Dyk, D. A., A Bayesian analysis of the multinomial probit model using marginal data augmentation. Journal of econometrics, 124(2)(2005) 311-334.

[20] Lawrence, E., Bingham, D., Liu, C. and Nair, V. N., Bayesian inference for multivariate ordinal data using parameter expansion. Technometrics, 50(2)(2008) 182-191.

[21] Leon, D., Alexander, R. and Carrigre, K. C., General mixed data model: Extension of general location and grouped continuous models. Canadian Journal of Statistics, 35(4)(2007) 533-548.

[22] Li, Y. and Schafer, D. W., Likelihood analysis of the multivariate ordinal probit regression model for repeated ordinal responses. Computational Statistics \& Data Analysis, 52(7)(2008) 3474-3492.

[23] Little, R. J. and Rubin, D. B., Statistical analysis with missing data. (John Wiley and Sons, New York, 2002).

[24] Liu, C. and Rubin, D. B., Ellipsoidally symmetric extensions of the general location model for mixed categorical and continuous data. Biometrika, 85(3)(1998) 673-688.

[25] Liu, X. and Daniels, M. J., A new algorithm for simulating a correlation matrix based on parameter expansion and reparameterization. Journal of Computational and Graphical Statistics, 15(4)(2006) 897-914.

[26] Liu, X., Daniels, M. J. and Marcus, B., Joint Models for the Association of Longitudinal Binary and Continuous Processes With Application to a Smoking Cessation Trial, Journal of the American Statistical Association, 104(2009) 429-438.

[27] McCulloch, R. E., Polson, N. G. and Rossi, P. E., A Bayesian analysis of the multinomial probit model with fully identified parameters. Journal of Econometrics, 99(1)(2000) 173-193.

[28] McCulloch, R. and Rossi, P., An exact likelihood approach to analysis of the MNP model. Journal of Econometrics, 64(1-2)(1994) 207-240.

[29] McFadden, D., Conditional logit analysis of qualitative choice behavior. In Frontiers of Econometrics, ed. by P. Zarembka. Academic Press(1973) 105-142.

[30] Nandram, B. and Chen, M. H., Reparameterizing the generalized linear model to accelerate Gibbs sampler convergence. Journal of Statistical Computation and Simulation, 54(1-3)(1996) 129-144.

[31] Olkin, I. and Tate, R. F., Multivariate correlation models with mixed discrete and continuous variables. The Annals of Mathematical Statistics(1961) 448-465.

[32] Peng, Y., Little, R. J. and Raghunathan, T. E., An extended general location model for causal inferences from data subject to noncompliance and missing values. Biometrics, 60(3)(2004) 598-607.

[33] Poon, W. Y. and Lee, S. Y., Maximum likelihood estimation of multivariate polyserial and polychoric correlation coefficients. Psychometrika, 52(3)(1987) 409-430.

[34] Schafer, J. L., Analysis of incomplete multivariate data. (CRC press, London, 1997).

[35] Snell, E. J., A scaling procedure for ordered categorical data. Biometrics, 20(1964) 592-607.

[36] Spiegelhalter, D. J., Best, N. G., Carlin, B. P. and Van Der Linde, A., Bayesian measures of model complexity and fit. Journal of the Royal Statistical Society: Series B (Statistical Methodology), 64(4)(2002) 583-639. 
[37] Tanner, M. A. and Wong, W. H., The calculation of posterior distributions by data augmentation. Journal of the American statistical Association, 82(398)(1987) 528-540.

[38] Teimourian, M., Baghfalaki, T., Ganjali, M. and Berridge, D., Joint modeling of mixed skewed continuous and ordinal longitudinal responses: a Bayesian approach. Journal of Applied Statistics, 42(10)(2015) 1-24.

[39] Van Dyk, D. A. and Meng, X. L., The art of data augmentation. Journal of Computational and Graphical Statistics, 10(1)(2001) 1-50.

[40] Xu, H. and Craig, B. A., A probit latent class model with general correlation structures for evaluating accuracy of diagnostic tests. Biometrics, 65(4)(2009) 1145-1155.

[41] Zhang, X., Boscardin, W. J., Belin, T. R., Wan, X., He, Y. and Zhang, K., A Bayesian method for analyzing combinations of continuous, ordinal, and nominal categorical data with missing values. Journal of Multivariate Analysis, 135(2015) 43-58. 